\title{
Percepción del paciente sobre calidad del cuidado humanizado brindado por la enfermera en una clínica-Lima
}

\author{
Haida Echevarría Schmidt, ${ }^{1, a, b, c}$
}

Echevarría SH. Percepción del paciente sobre calidad del cuidado humanizado brindado por la enfermera en una clínica-Lima. Cuid salud, ene-jun 2017; 3(1):239-248.

\section{RESUMEN}

En las últimas décadas el estudio del cuidado humanizado ha sido objeto de creciente interés. Cabe al equipo de salud proporcionar cuidado holístico con calidad y calidez, principalmente a enfermería por su permanencia con el paciente. Objetivo: determinar la percepción del paciente sobre el cuidado humanizado que brinda la enfermera en los servicios de hospitalización de la Clínica Tezza. Metodología: estudio de enfoque cuantitativo y diseño descriptivo; cuya muestra fue 250 pacientes de 20-70 años de edad elegidos mediante criterio probabilístico. Los datos se recolectaron utilizando la encuesta y un instrumento basado en "percepción de comportamientos de cuidado humanizado de enfermería" (PCHE). el análisis de datos fue mediante la estadística descriptiva en programa SPSS versión 21.0. Resultados: en la percepción del cuidado humanizado global, el $84 \%$ de pacientes la calificó como excelente. En la correlación por dimensiones la edad se correlacionó con hacer enfermero $(\mathrm{p}=0,020)$ y la proactividad $(\mathrm{p}=0,035)$; el sexo con hacer enfermero $(\mathrm{p}=0,001)$, apoyo físico $(\mathrm{p}=0,037)$ y empatía $(\mathrm{p}=0,033) \mathrm{y}$, el tiempo de hospitalización con empatía $(\mathrm{p}=0,031)$. Conclusiones: la percepción del cuidado humanizado en los pacientes de la clínica fue considerada excelente, correlacionándose principalmente con edad menor 40 años, sexo femenino y una hospitalización de 2-5 días.
Echevarría SH. Perception of the patient about humanized care provided by the nurse in a Clinic-Lima. Cuid salud, ene-jun 2017; 3(1):239-248.

\begin{abstract}
In the last decades the study of humanized care has been an object of growing interest. It is up to the health team to provide holistic care with quality and warmth, mainly to nursing because of its permanence with the patient. Objective: To determine the patient's perception of the humanized care provided by the nurse in the hospitalization services of Clínica Tezza. Methodology: Study of quantitative approach and descriptive design; whose sample was 250 patients of 20-70 years of age chosen by means of probabilistic criteria. Data were collected using the survey and an instrument based on "perception of humanized nursing care behaviors" (PCHE). The data analysis was done using descriptive statistics in SPSS v. 21.0. Results: In the perception of global humanized care, $84 \%$ of patients rated it as excellent. In the correlation by dimensions, age was correlated with nursing $(\mathrm{p}=0.020)$ and proactivity $(\mathrm{p}=$ $0.035) ;(p=0.037)$ and empathy $(p=0.033)$, and the time of hospitalization with empathy $(\mathrm{p}=0.031)$. Conclusions: The perception of the humanized care in the patients of the Clinic was considered excellent, being related mainly with age younger than 40 years, female sex and a hospitalization of 2-5 days.
\end{abstract}

Keywords: Perception, nursing care, humanization of assistance, patient satisfaction (Source: MeSH NLM).

Palabras clave: percepción, cuidado de enfermería, humanización de la asistencia, satisfacción del paciente (Fuente: DeCS BIREME).

\footnotetext{
1 Escuela de Enfermería Padre Luis Tezza afiliada a la Universidad Ricardo Palma, Lima-Perú.

a Magister en Teología.

b Licenciada en Enfermería.

Religiosa de la Congregación Hijas de San Camilo.
} 


\section{INTRODUCCIÓN}

La Organización Mundial de la Salud (OMS) afirma que toda persona tiene derecho al más alto nivel de salud alcanzable, incluyendo la calidad de su atención como un objetivo del macro proyecto salud para todos, que comprende la percepción y satisfacción del paciente. $^{1}$ Aspecto destacado por la Joint Commission International (JCI) al solicitar a las instituciones coordinar sus actividades que garanticen la calidad dentro de un programa institucional integrado. ${ }^{2}$

Calidad que debe cuidar no solo la esfera biológica y sintomática del paciente, sino también aquellas esferas subjetivas, emotivas, espirituales y culturales. En ese sentido, enfermería históricamente ha orientado su servicio al cuidado holístico, con sensibilidad y responsabilidad ética valorando la dignidad humana de cada ser.

Aunque, Poblete y Valenzuela, ${ }^{3}$ citando a Foucault, refieren que las instituciones hospitalarias han impuesto desde finales del siglo XVIII una atención centrada en la patología y en el saber médico, dejando atrás la individualidad e integridad del enfermo; visión que se consolida en los siglos posteriores y que aún se reproduce en muchos hospitales bajo el modelo biomédico y curativo, fuertemente estructurado en los sistemas de salud actuales, que ha conllevado a los profesionales focalizar su atención más en la ejecución de procedimientos y técnicas, normativas y roles, que en el cuidado al propio paciente, de su entorno y familia.

Becerril $^{4}$ refiere que los usuarios, la familia y la sociedad en su conjunto aluden un trato mecanicista, despersonalizado y con ausencia de empatía personal y profesional por parte de la enfermera. Caracterizando al cuidado institucionalizado con falta de continuidad en el proceso, atención multiprofesional, masificación y despersonalización de la atención en salud, lo que refleja un proceso de cuidado deshumanizado. ${ }^{5}$

Según Watson, una causa de la deshumanización sería la reestructuración administrativa ocurrida en los sistemas sanitarios del mundo, siendo necesario ir al rescate del aspecto humano, espiritual y transpersonal, ${ }^{6}$ principalmente en el área asistencial. Aunque en esta área muchas veces es difícil conservar los valores humanitarios, además de verse las enfermeras invisibilizadas debido a las múltiples actividades que realizan. ${ }^{3}$

Mismo así, el cuidado debe estar impregnado de valores, voluntad y compromiso para cuidar, conocimiento, acciones de cuidado y consecuencias; ${ }^{7}$ desarrollado para satisfacer las necesidades del ser humano con el propósito de promover, mantener o recuperar su salud; resaltando los valores altruistas como la amabilidad, empatía, preocupación y amor por uno mismo y por los otros. Si la enfermera es capaz de ser sensible consigo misma, será sensible con los demás, demostrando interés por sus problemas y fortaleciendo el desarrollo del otro, estableciendo una relación transpersonal e intersubjetiva, de enseñanzaaprendizaje, respetando las creencias, la fe y esperanza del paciente. ${ }^{8}$

De este modo, se hace imperativo retomar el cuidado desde su dimensión mística, fortaleciendo la capacidad de comunicación y escucha, de respeto por las creencias del otro, con verdadera entrega, compromiso y calidez. Marcando la diferencia entre atención de enfermería y cuidado humanizado, trascendiendo la realidad, donde lo espiritual, la visión de vida, las realidades importantes y las metas del paciente son relevantes. $^{9}$

En el ámbito internacional, los estudios reportan que los pacientes perciben que las enfermeras brindan un cuidado humanizado casi siempre o siempre; ${ }^{7,10-12}$ destacado la consideración a los sentimientos, el apoyo físico y las cualidades de la enfermera; ${ }^{10,11}$ el apoyo emocional, ${ }^{11}$ la confianza, la amabilidad y el trato personalizado. ${ }^{12}$ Contrarios a los reportados en el Perú, donde la percepción del cuidado va desde medianamente favorable ${ }^{13}$ a baja calidad; principalmente por la escaza disponibilidad para la atención, empatía, apoyo emocional y respeto a los sentimientos del paciente. ${ }^{14}$ Situación que muestra la pérdida de valores altruistas en el cuidado cotidiano, bien como la escasa información derivados de estudios de pequeño porte.

Hechos que propician la realización del estudio en la Clínica Tezza en Lima ciudad, institución que pertenece a la Congregación Religiosa Hijas de San Camilo de Lellis y que tiene como misión Cuidar al enfermo como lo hace una madre amorosa con su único hijo enfermo. ${ }^{15}$ Lema que es supervisado y controlado desde la instancia administrativa de la gerencia, por las hermanas religiosas de la Congregación.

La Clínica Tezza es una Empresa Prestadora de Servicios de Salud-EPS que tiene convenios con instituciones del Estado e instituciones privadas desde hace más 20 años, ofreciendo servicios en todas las especialidades y grupos etarios, desde los programas de promoción de la salud / prevención de la enfermedad, 
hasta unidades de cuidados intensivos altamente especializados y cuidados paliativos, cuyo personal biomédico es preferencialmente laico. Entre las enfermeras, un porcentaje minoritario pertenece a la Congregación y desempeña básicamente labor administrativa, además realiza la inducción al carisma de San Camilo a todo personal recién ingresado acorde con la misión institucional y, que eventualmente, suplen a las enfermeras asistenciales cuando faltan.

En sus 45 años de fundación se desconoce de forma objetiva qué tipo de cuidado se brinda a los pacientes en la Clínica, especialmente porque enfermería está pendiente de sus requerimientos y cuidados las 24 horas del día; a fin de reorientarse las políticas institucionales y/o difundirlo como modelo de cuidado. En tal sentido, el objetivo del estudio fue determinar la percepción del paciente sobre la calidad del cuidado humanizado brindado por la enfermera en los servicios de hospitalización de la Clínica Tezza y correlacionarlos con las variables sexo, edad y tiempo de hospitalización.

\section{METODOLOGÍA}

El estudio fue de enfoque cuantitativo, de método no experimental y diseño descriptivo correlacional, de corte transversal.

El área fue la Cínica Padre Luis Tezza, comúnmente denominada Clínica Tezza, sito en el distrito de Santiago de Surco Lima-Perú; institución dirigida por la Congregación Hijas de San Camilo, que busca brindar una atención personalizada que satisfaga las necesidades de salud integral de los pacientes, haciendo realidad el mandato de Cristo: cuidar con amor de madre a los que sufren, concretizando el deseo del Fundador San Camilo, estar siempre dispuesto a hacer cualquier sacrificio por amor al enfermo. ${ }^{15}$

La Clínica dispone de más de 25 servicios especializados para cubrir las necesidades de los pacientes y atiende a personas con derechohabiente, según legislación peruana, ${ }^{16}$ acogiendo a peruanos de los distintos estratos sociales mediante atención particular o por convenios de salud firmadas con diversas instituciones. Siendo el área elegida los servicios de hospitalización ubicados en el segundo piso del predio, donde se internan pacientes de medicina, cardiología, endocrinología, cirugía, oncología, entre otros.

Esta institución cuenta con 250 profesionales enfermeras de nivel universitario, quienes brindan servicio de cuidado asistencial las 24 horas del día. Del total de enfermeras, solo el $5 \%$ pertenece a la Congregación Religiosa Hijas de San Camilo y básicamente ocupan cargos administrativos de enfermería en la Clínica, gestionando los recursos materiales y humanos para brindar un mejor servicio.

La población fue de 710 pacientes hospitalizados en el último trimestre del 2015, según datos de la Oficina de Estadística de la Clínica. De ese total, se determinó la muestra $(n=250)$ utilizando la fórmula de población finita y los valores de prevalencia de estudios previos, elegidos mediante muestreo probabilístico aleatorio simple; considerando los siguientes criterios de inclusión: personas de ambos sexos, con edades entre 20 y 70 años, hospitalizados más de dos días, participación voluntaria, orientados en tiempo, espacio y lucidos. Y de exclusión: pacientes extranjeros, miembros de alguna congregación religiosa y con problemas psiquiátricos.

El instrumento fue un cuestionario estructurado compuesto de título, instructivo, datos generales y aspectos relacionados con la calidad del cuidado, basado en la primera versión del instrumento "Percepción de Comportamientos de Cuidado Humanizado de Enfermería" (PCHE) de Rivera y Triana; ${ }^{17}$ del cual fue omitido la categoría "Características de la enfermera" (ítems 2, 5, 7, 8, 9 y 46) y los ítems 16, 31, 32 y 49 por considerarlos reiterativas, adicionándose a su vez las categorías "Apoyo espiritual" y "Promoción de la enseñanza aprendizaje" acorde con la propuesta CARITAS del cuidado humanizado de Watson. ${ }^{18}$

Con esta adaptación y complementación el instrumento quedó conformado por 10 categorías, conservando el número de 50 ítems con 5 ítems en cada categoría y cinco opciones de respuestas medidas a través de una escala de estimación: $1=$ nunca, $2=$ a veces, $3=$ casi siempre, $4=$ siempre, como propuesto en el PCHE original. ${ }^{17}$ En este formato, el instrumento fue sometido a prueba de validez mediante juicio de expertos y analizados con la prueba Binomial ( $p=0,041)$; en esta fase, apenas fueron adecuados unos pocos ítems al contexto cultural local. Luego, fue desarrollada una prueba piloto para la confiabilidad de los ítems, resultados que fueron analizados con la prueba Alfa de Chronbach $(\alpha=0,98)$, identificándose alta correlación entre los mismos.

En la calificación del instrumento, primero se obtuvieron la media y desviación estándar; además de proporciones según puntuación: excelente (164-200 puntos), bueno (126-163 puntos), aceptable (88-125 
puntos) y malo (50-87), con valores máximos y mínimos. Cada categoría oscila entre 17-20 puntos para "excelente" y de 5-8 puntos en "malo".

La variable "Percepción de la calidad del cuidado humanizado" fue definida como el juicio o interpretación del paciente sobre el cuidado que recibe de la enfermera involucrando sus necesidades biológicas y de tratamiento con pericia y asertividad, además de sus necesidades emocionales, socioculturales y espirituales, demostrando una actitud de disponibilidad, receptividad, proactividad, escucha, confianza y reciprocidad; evitando en lo posible el daño y el malestar, que facilita su crecimiento humano y ontológico, respetando sus creencias culturales y espirituales.

Para la recolección de datos se cumplió con los procedimientos administrativos previamente. Concluida esta fase, la investigadora recolectó los datos entre febrero y abril de 2016 en el horario de 14.00-18.00 horas; la elección de los pacientes fue realizada mediante el uso de la tabla randomica con reposición, cuando el paciente no cumplía con los criterios de inclusión, cuya verificación era constatada en la historia clínica.

Luego, se abordaba a cada paciente en su habitación, explicando el propósito del estudio y sus beneficios: obtenida su aquiescencia en la Hoja de Consentimiento Informado se aplicaba la encuesta, brindando todo el apoyo necesario con la finalidad de esclarecer y responder a más dudas e inquietudes.

Para el análisis de la información recolectada, los datos fueron codificados en una base del programa Excel 2013; luego exportados al paquete estadístico SPSS v. 21.0 para procesar el análisis haciendo uso de estadígrafos, además de la prueba de Estaninos para determinar la clasificación de la calidad del cuidado humano y por cada categoría. Para correlacionar las variables se utilizó la prueba de Chi cuadrado de Pearson y prueba de Spearman, considerando un $p$ de 0,05 como significativo.

El proyecto del estudio fue evaluado por el Comité de Ética en Investigación de la Facultad de Medicina Humana de la Universidad Ricardo Palma, siendo aprobado mediante oficio $\mathrm{N}^{\circ}$ 0802015/CARD AB; teniendo en cuenta los principios fundamentales aplicados en la investigación con seres humanos.

\section{RESULTADOS}

Tabla 1: Datos de pacientes hospitalizados. Clínica Tezza, febrero-abril 2016.

\begin{tabular}{llrr}
\hline DATOS & CATEGORÍA & n=250 & $\mathbf{1 0 0 \%}$ \\
\hline Edad & 20 a 29 años & 81 & 32,4 \\
& 30 a 39 años & 48 & 19,2 \\
& 40 a 49 años & 56 & 22,4 \\
50 a 59 años & 28 & 11,2 \\
Sexo & 60 años a más & 37 & 14,8 \\
Días hospitalizados & Masculino & 104 & 41,6 \\
& Femenino & 146 & 58,4 \\
& De 2-5 días & 209 & 83,6 \\
& De 6 a más & 41 & 16,4 \\
\hline
\end{tabular}

En la tabla 1, la mayoría de pacientes tenía edades entre 20-49 años, predominando las personas de sexo femenino, con un periodo de hospitalización de 2-5 días.

Gráfico 1: Calidad del cuidado humanizado global de la enfermera percibido por el paciente. Clínica Tezza, febrero-abril 2016.

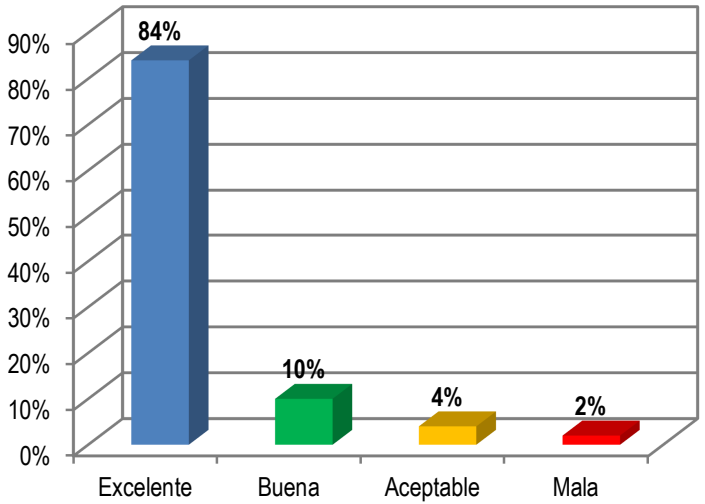

Tabla 2: Categorías de la calidad del cuidado humanizado de la enfermera percibido por el paciente. Clínica Tezza, febrero-abril 2016.

\begin{tabular}{lrr}
\hline Categorías del cuidado humanizado & $\overline{\mathbf{x}}$ & $\mathbf{\pm D S}$ \\
\hline 1. Sentimientos del paciente & 19,8 & 3,5 \\
2. Del hacer de la Enfermera & 19,0 & 3,6 \\
3. Proactividad & 18,5 & 3,8 \\
4. Apoyo emocional & 18,7 & 3,7 \\
5. Apoyo físico & 18,6 & 3,8 \\
6. Empatía & 18,6 & 4,1 \\
7. Priorizar al ser del cuidado & 18,6 & 3,6 \\
8. Disponibilidad para el cuidado & 18,4 & 3,9 \\
9. Apoyo espiritual & 18,3 & 4,0 \\
10. Promoción de la enseñanza-aprendizaje & 18,0 & 4,2 \\
\hline
\end{tabular}


Respecto a la percepción de la calidad del cuidado humanizado por el paciente, en todas las dimensiones las medias alcanzaron una apreciación "excelente"; con la media más alta en la dimensión "sentimiento del paciente" y más baja en "apoyo espiritual" y "promoción de enseñanza-aprendizaje" (tabla 2).

Tabla 3: Relación entre sexo del paciente y percepción de la calidad del cuidado humanizado según categorías y global. Clínica Tezza, febrero-abril 2016.

\begin{tabular}{llr}
\hline Categorías del cuidado & Valor de $\boldsymbol{p}^{*}$ \\
\hline 1. Sentimientos &, 907 \\
2. Del hacer e la enfermera &, 001 \\
3. Proactividad &, 217 \\
4. Apoyo emocional &, 883 \\
5. Apoyo físico &, 037 \\
6. Empatía &, 033 \\
7. Priorizar &, 512 \\
8. Disponibilidad &, 528 \\
9. Apoyo espiritual &, 661 \\
10. Promoción de la salud &, 933 \\
11. Calidad cuidado humanizado &, 419 \\
\hline
\end{tabular}

*Chi cuadrado de Pearson

Tabla 4: Relación entre edad $<40 \geq$ años del paciente y percepción de la calidad del cuidado humanizado. Clínica Tezza, febrero-abril 2016.

\begin{tabular}{lcc}
\hline Categorías del cuidado & $\begin{array}{c}\text { Coeficiente de } \\
\text { correlación }\end{array}$ & $\begin{array}{c}\text { Valor de } \\
\boldsymbol{p}^{* *}\end{array}$ \\
\hline 1. Sentimientos &, 093 &, 143 \\
2. Del hacer de la enfermera &, 147 &, 020 \\
3. Proactividad &, 134 &, 035 \\
4. Apoyo emocional &, 098 &, 122 \\
5. Apoyo físico &, 049 &, 443 \\
6. Empatía &, 072 &, 256 \\
7. Priorizar &, 043 &, 500 \\
8. Disponibilidad &, 091 &, 152 \\
9. Apoyo espiritual &,- 002 &, 979 \\
10. Promoción de la salud &,- 035 &, 578 \\
11. Calidad cuidado humanizado &, 092 &, 145 \\
\hline
\end{tabular}

**Prueba de Spearman

Tabla 5: Relación entre tiempo $<6 \geq$ días de hospitalización del paciente y percepción de la calidad del cuidado humanizado. Clínica Tezza, febrero-abril 2016.

\begin{tabular}{|c|c|c|}
\hline $\begin{array}{l}\text { Categorías del cuidado } \\
\text { humanizado }\end{array}$ & $\begin{array}{l}\text { Coeficiente de } \\
\text { correlación }\end{array}$ & $\begin{array}{c}\text { Valor de } \\
p^{* * * *}\end{array}$ \\
\hline 1. Sentimientos &,- 059 & ,356 \\
\hline 2. Del hacer de la enfermera &,- 067 & ,295 \\
\hline 3. Proactividad &,- 058 & ,360 \\
\hline 4. Apoyo emocional &,- 080 & ,206 \\
\hline 5. Apoyo físico &,- 114 & ,078 \\
\hline 6. Empatía &,- 136 & 031 \\
\hline 7. Priorizar &,- 116 & 067 \\
\hline 8. Disponibilidad &,- 011 & ,865 \\
\hline 9. Apoyo espiritual &, 030 & 634 \\
\hline 10. Promoción de la salud &,- 099 &, 118 \\
\hline $\begin{array}{l}\text { 11. Calidad del cuidado } \\
\text { humanizado }\end{array}$ &,- 084 &, 186 \\
\hline
\end{tabular}

\section{DISCUSIÓN}

El cuidado de enfermería es un conjunto de acciones que busca la sanación y el bienestar del ser cuidado, lo que implica ser facilitadores de la satisfacción de necesidades, generando una relación interpersonal enfermera-paciente y fomentando la conservación de la salud y la recuperación de su equilibrio holístico: físico, social, mental y espiritual. ${ }^{19}$

De esta manera, el cuidado humanizado de enfermería debe caracterizarse por el trato personalizado y holístico, entendiendo que "el otro" necesita ser cuidado en la totalidad de su persona y no apenas en lo biológico; lo cual únicamente será posible lograrlo con una respuesta de trabajo en equipo y centrado en la persona. $^{20}$ Perspectiva que constituye un reto para el profesional de enfermería, ante la realidad deshumanizante que se vive en el mundo actual.

Los resultados del estudio muestran que los pacientes en la Clínica Tezza perciben que las enfermeras brindan cuidado humanizado de modo general (gráfico 1), siendo en su mayoría las personas de sexo femenino, viviendo la etapa de la adultez joven y madura y con un periodo de hospitalización de 2-5 días (tabla 1). Datos que se asemejan a resultados previos, ${ }^{10,11}$ aunque los pacientes tuvieron en esos estudios mayores días de hospitalización.

Por otro lado, los pacientes calificaron la calidad del cuidado humanizado como excelente (gráfico 1), apreciación que se evidencia también en el análisis por categorías donde las medias obtenidas están en el rango de 17-20 puntos (tabla 2). Resultados que concuerdan con los presupuestos de Watson, demostrando que el cuidado es el eje central de la enfermería y que se 
ejecuta mediante vinculo enfermera-paciente, que va más allá de las habilidades técnicas y del conocimiento científico, abarcando las cualidades del ser en esencia. ${ }^{19}$

El estudio de Miranda et al., ${ }^{11}$ realizado en un Hospital Base de Valdivia-Chile y de Borré, ${ }^{12}$ realizado en un hospital de Barranquilla-Colombia muestran resultados semejantes. A su vez, Bautista et al., ${ }^{10}$ refieren que los pacientes están conformes con el cuidado humanizado que reciben en hospitales de III y IV nivel-Colombia. En el ámbito nacional se reporta una percepción medianamente favorable ${ }^{13,21}$ y medianamente desfavorable sobre el cuidado; ${ }^{22}$ llegando a calificarla como de baja calidad porque no se prioriza el cuidado. ${ }^{13}$ Características que suelen ser común en las instituciones públicas del país, posiblemente porque la relación enfermera-paciente supera las recomendaciones establecidas.

En el análisis por categorías, los sentimientos del paciente presentan la más alta media en el cuidado humanizado proporcionado por la enfermera (tabla 2). Lo que refleja el carisma de las enfermeras que laboran en la Clínica, haciendo alusión a su lema "cuidar al enfermo como lo hace una madre amorosa con su único hijo enfermo"; trascendiendo la visión reduccionista de cumplir solo con los procedimientos y la administración de medicamentos.

Una posible explicación de este hecho sería la insistencia que hace la Congregación para cumplir con el lema, donde es relevante la inducción al personal nuevo, la supervisión de las enfermeras religiosas a las enfermeras laicas, recordando el carisma y retroalimentando el carácter humanístico y espiritual que las caracteriza de otros hospitales o clínicas privadas. Rivera y Triana, ${ }^{17}$ destacan desde la teoría de Watson, que la persona es un ser integral, con capacidad de participar en el planeamiento y ejecución de su cuidado, valorando los sentimientos que emanan de la interacción enfermeras-paciente, como el apoyo, acogida y atención. Cuidado que debe trasmitir afecto, ayuda para crecer como ser humano, totalmente saludable y que favorece la pronta recuperación porque mejora el sistema de comunicación y de contacto, incidiendo directamente en el bienestar del paciente. $^{23}$

Contrario a lo esperado, las categorías menos evaluadas fueron promoción de la enseñanzaaprendizaje y apoyo espiritual (tabla 2), mostrando variabilidad en la percepción de los pacientes; siendo elemental fomentar la educación del paciente para desarrollar acciones de autocuidado, más aún si se incorpora a la familia, que convive con él y que le dará soporte en su recuperación en el hogar. Compete a la enfermera aclarar las dudas y brindar reiteradas explicaciones sobre su cuidado si el paciente lo requiere, con conocimiento y seguridad en el acto de cuidado haciendo gala de expresiones de interés, consideración, respeto y sensibilidad en sus palabras, tono de voz, actitud, gestos y modos de tocar. ${ }^{18}$ Watson refiere que la verdadera expresión del arte y ciencia del cuidado holístico conjuga conocimiento, habilidades, experiencia, intuición y expresión de la sensibilidad. ${ }^{8}$

Sobre el apoyo espiritual, Pancrazzi ${ }^{24}$ acota que esta acción proporciona alivio en los pacientes, sobre todo, en los momentos de sufrimiento y, fortaleza en la debilidad. Afirma la vida y considera la agonía como un proceso normal, sin pretender alargar la vida a toda costa ni acelerar la muerte; brindar apoyo espiritual incluye integrar a la familia para que juntos puedan sobrellevar la enfermedad y el propio sufrimiento.

Según Watson, ${ }^{8}$ existen misterios y aspectos filosóficos y metafísicos de la experiencia humana que no se ajustan a la ciencia y al pensamiento racional; sin embargo, son reales y están llenos de significados espirituales que emergen abierto a infinitas posibilidades de milagros, basados en las creencias culturales, mitos y metáforas y vida subjetiva del mundo interno, tanto de las enfermeras y los pacientes, como de sus familias, permitiendo muchas veces las curas y curaciones milagrosas. Significados que muchas veces están negligenciados en el momento del cuidado, debiendo revalorarse su contribución al pleno bienestar del paciente y familia, aun en los momentos en que todo parece acabar con la vida.

La espiritualidad mística se revela como experiencia intima, profunda y personal, que se traduce en el trato humanizado y holístico que debe caracterizar a la enfermera. La mística, en tanto que experiencia del misterio, la religiosidad y la entrega, concierne a la vida en sí misma; es la devoción y la apertura al misterio con la que una persona realiza su trabajo en esencia, expresando la calidad y el sentido de entrega, arraigado en sus creencias. ${ }^{25}$ Entre tanto, desarrollar la mística en las enfermeras es un reto ante la deshumanización del cuidado en el mundo de hoy. Alarcón ${ }^{9}$ señala que las personas tienen necesidad de amar y ser amadas, necesidad de decir adiós, necesitando ser reconfortados en su fe y prácticas religiosas. Hecho que diferencia entre la atención de enfermería y el cuidado humanizado de una enfermera con mística. ${ }^{3}$

En el estudio fueron incluidos los pacientes de todas las áreas de atención, entre ellos los de oncología, cardiología, traumatología, postoperados, medicina, 
entre otros. Es posible que, en esta diversidad de condiciones, la esperanza y la fe emergieran como cimientos fundamentales de la existencia humana, aunque posiblemente no con la misma intensidad en todos los casos, por eso las medias muestran resultados menores a los alcanzados en otras categorías; siendo más crítica su vivencia y por lo tanto su cuidado, en pacientes que atraviesan momentos de enfermedad grave, dolor intenso y ante la muerte.

En la asociación de la calidad de cuidado humanizado con las variables sexo, edad y tiempo de hospitalización se identifica que no existe correlación significativa con ninguna de esas variables (tabla 3, tabla 4 y tabla 5). Concordando con los estudios de Romero et al. ${ }^{26}$ y Miranda et al. ${ }^{11}$

Sin embargo, algunas de las categorías de la calidad del cuidado humanizado fueron correlacionados con el sexo femenino: hacer de la enfermera, empatía y apoyo físico (tabla 3). Lo que puede significar que las mujeres son más sensibles y atentas a estos comportamientos en el cuidado. Siendo que el hacer de la enfermera evidencia su actuar como profesional, considerando que su principal objetivo es asistir integralmente a la persona enferma; acciones que se fundamentan en el conocimiento científico y en los principios bioéticos, además de habilidades y destreza que ofrecen seguridad y confianza al paciente. Sobre la empatía, Romero-Massa et al., ${ }^{7}$ citando a Rogers, refieren que es uno de los elementos básicos para trabajar con el otro en una relación de ayuda. Es decir, considerando sus preocupaciones, sufrimientos, anhelos y aspiraciones, permitiéndole expresar los sentimientos sobre su enfermedad y tratamiento, estableciendo una relación agradable, de escucha atenta y colocándose en el lugar del otro. Finalmente, el apoyo físico demuestra la importancia del contacto físico, tacto delicado y sutil, preservando siempre la dignidad del otro; satisfaciendo las necesidades físicas, manejando el dolor y proporcionando comodidad física. ${ }^{17}$

Características que reflejan una persona cálida, querida, especial, agradable, simpática, amable y cordial en el cuidado; eficiente en su desempeño y clara en la comunicación, que manifiesta estímulos positivos y brinda cuidado comprometido y solidario. ${ }^{18}$ Comportamiento que revela la naturaleza ideal de una enfermera, identificada con su condición humana y humanista; lejos de lo que actualmente se conocen en los sistemas de salud; atrapados en los discursos dominantes como la tecnología, la enfermedad y la práctica basada en evidencia. ${ }^{19}$
Respecto a la edad, tener $\leq 39$ años se correlacionó también con del hacer de la enfermera y con la proactividad (tabla 4); sin embargo, se observa una correlación baja en ambos comportamientos de la enfermera $\quad(\mathrm{r}=0,14 ; \mathrm{r}=0,13$ respectivamente). La proactividad se refiere a la iniciativa que tienen las enfermeras para realizar sus actividades conducentes a un mejor desempeño; aspecto que preconiza la identificación de las necesidades del paciente en toda su integralidad, ofreciendo respuestas, instruyendo sobre su cuidado e informándole de todo lo necesario para que tome las mejores decisiones sobre su salud y cuidado. ${ }^{17}$ Estos resultados muestran que la población joven en el estudio identifica estas dos cualidades del cuidado humanizado como relevante.

Cuanto al tiempo de hospitalización los resultados muestran una correlación negativa con respecto a la categoría empatía, lo que significa que a menor tiempo de hospitalización mayor posibilidad de empatía por parte de las enfermeras hacia los pacientes (tabla 5). Relación que al mismo tiempo expresa que, cuanto mayor sea los días de hospitalización la actitud para el cuidado puede tornarse posiblemente neutra o apática.

El estudio de Romero et al. ${ }^{26}$ reporta que el tiempo de hospitalización no se correlaciona con el cuidado humanizado, destacando que las autoras consideraron un periodo de hospitalización de semanas y mayor de dos semanas. Asimismo, el estudio de Miranda et al., ${ }^{11}$ cuya media de tiempo de hospitalización fue de 6,95 días tampoco mostró correlación en estas variables. Corroborando que a mayor tiempo de hospitalización menor calidad en el cuidado humanizado.

Hecho que debe preocupar al sistema sanitario y principalmente, a los gestores en salud de la Clínica y de los hospitales, ya que este componente intangible en salud constituye un indicador de la satisfacción en los sistemas de salud, siendo relevante para fortalecer y mejorar los factores que determinan la calidad de la atención. ${ }^{12}$ Tal parece que la buena atención y cuidado dura poco tiempo, mientras tanto, el cuidado lleno de carisma y de valores altruistas debe ser permanente. Watson reconoce que el cuidar es parte fundamental del ser y es el acto más primitivo que un ser humano realiza para efectivamente llegar a ser, existiendo en relación con el otro; en enfermería este cuidado es mucho más que eso, es profesional, estructurado, formalizado y destinado a satisfacer al ser humano. ${ }^{19}$

En concordancia con Collière, Romero-Massa et al. ${ }^{7}$ destacan que las enfermeras en los servicios sanitarios terminan realizando técnicas y tareas cada vez más especializadas, sobrecargándose de labores 
dependientes e interdependientes de la disciplina médica. Afirmación que toma mucha más relevancia en este milenio, donde la introducción de máquinas y procedimientos altamente sofisticados hacen que ellas soslayen la relación de cuidado humanizado con el paciente.

Torres ${ }^{27}$ refiere que el cuidado de enfermería está orientado a priorizar la persona, con preocupación por ella y respeto por sus decisiones e intimidad, donde la calidad del cuidado se evidencia a partir de saber identificar al paciente por su nombre. Desde la visión de Watson, el objetivo de la relación transpersonal de cuidado corresponde a proteger, realzar, y conservar la dignidad de la persona, la humanidad, la integridad y la armonía interior. ${ }^{8}$

De acuerdo con los resultados del estudio, las enfermeras en la Clínica Tezza ofrecen un cuidado humanizado en general, calificado como excelente, brindando prioridad a los sentimientos del paciente, desempeñando su hacer con destreza, calidez y afectividad dando soporte a sus necesidades emocionales, con la esencia mística de la enfermera, tal y como lo relatan Sosa-Rosas y Cuamatsi-Peña, ${ }^{28}$ tratándose de una profesión humana que implica considerar la espiritualidad de la persona. Realidad que contrasta mucho con otros hospitales del servicio público en el ámbito nacional, en donde es necesario dotar de mayor recurso humano y desarrollar acciones de sensibilización para humanizar la asistencia y el cuidado en todo el personal de salud, a fin de rescatar la mística esencial de cada profesión, haciendo gala de generosidad, responsabilidad y entrega. ${ }^{25}$ Proceso que implica la participación de los gestores y líderes políticos en salud y especialmente, en la profesión de enfermería que siempre permanece más próximo del paciente por la naturaleza de su labor.

\section{Correspondencia:}

Haida Echevarría Schmidt

Correo electrónico: haida.schmidt@gmail.com

\section{REFERENCIAS BIBLIOGRÁFICAS}

1. Organización Mundial de Salud. Informe sobre la salud en el mundo 2001. Salud mental: nuevos conocimientos, nuevas esperanzas [internet]. Ginebra: OMS; 2001 [citado 9 ago 2014]. p.169. Disponible en http://www.who.int/whr/2001/en/whr01_es.pdf
Asimismo, es importante resaltar la necesidad de ofrecer el cuidado humanizado de forma regular y permanente, ya que el estudio demostró que a menor tiempo de hospitalización mayor empatía por parte de las enfermeras; siendo que el sexo femenino se correlaciona con del hacer de la enfermera, empatía y apoyo físico y, la menor edad con proactividad y del hacer de la enfermera. Comportamientos positivos que deben seguir promoviendo y promoverse en otros hospitales y clínicas privadas, entendiendo que el ser humano es un ser altamente complejo y que tiene vulnerado su capacidad de autosuficiencia y autopercepción humana cuando enfrenta una enfermedad y que el sistema de salud está para cuidarlo y protegerlo, favoreciendo su reintegración al sistema social y familiar sin menoscabo de su dignidad.

Una contribución del estudio respecto al instrumento PCHE es la adición de las categorías espiritualidad y enseñanza-aprendizaje, acorde con los 10 factores del proceso caritas de Watson, sin embargo, es necesario seguir comprobando su confiabilidad en posteriores estudios, a fin de dar mayor solidez y robustez al instrumento como un todo. Categorías que también deben ser mejor visualizadas en el trabajo del cuidado al enfermo, dado que la profesión de enfermería se compromete con el cuidado holístico integral, desde la esencia de potencialidades que tiene cada persona.

\section{Declaración de financiamiento y de conflictos de interés:}

El estudio fue financiado por la autora, declara no tener conflictos de interés.

\section{Contribución de auditoría:}

Echevarría SH: Concepción y diseño del estudio, recolección de los datos, análisis e interpretación de los resultados y aprobación de la versión que será publicada.

2. Joint Commission International (JCI). Estándares de acreditación para hospitales de Joint Commisssion International. Spanish 5ta edición [internet]. Oakbrook Terrace-Illinois-EEUU: JCI; 2014 [citado 6 ago 2016]. p.11. Disponible: https://www.jcrinc.com/assets/1/14/EBJCIH14S_Sa mple_Pages.pdf 
3. Poblete TM, Valenzuela SS. Cuidado humanizado un desafío para las enfermeras en los servicios hospitalarios. Acta Paul Enferm [serie de internet]. Sep 2007 [citado 18 may 2014]; 20(4): 499-503. Disponible en: http://www.scielo.br/pdf/ape/v20n4/18.pdf

4. Cárdenas BL. El humanismo en la formación y práctica de enfermería: una esperanza trasformadora. Texto Contexto Enferm [serie de internet]. 2016 [citado 10 oct 2016]; 25(1): [cerca 02 laudas]. Disponible en: http://www.scielo.br/pdf/tce/v25n1/es_0104-0707tce-25-01-editorial.pdf

5. Gonzales-Juárez L, Velandia-Mora AL, FloresFernández V. Humanización del cuidado de enfermería. De la formación a la práctica clínica. Rev CONAMED [serie de internet]. 2009 [citado 12 may 2016]; Supl:40-43. Disponible en: http://www.imbiomed.com/1/1/articulos.php?metho $\mathrm{d}=$ showDetail\&id_articulo=59710\&id_seccion=702 $\underline{\text { \&id_ejemplar }=6038 \& i d \_ \text {revista }=41}$

6. Watson J. Ponencia del Primer congreso internacional de Enfermería. Facultad de Enfermería y Nutriología. [Internet]. Chihuahua-México: Universidad Autónoma de Chihuahua [citado 20 mar 2014] Disponible en: http://www.fen.uach.mx/index.php?page/ $\underline{\text { Semblanza_Jean_Watson }}$

7. Romero-Massa E, Contreras-Méndez I, Pérez-Pájaro Y, Moncada A, Jiménez-Zamora V. Cuidado humanizado de enfermería en pacientes hospitalizados. Cartagena, Colombia. Rev cienc biomed [serie de internet]. Ene-jun 2013 [citado 24 ago 2015]; 4(1): 60-68. Disponible en: http://www.revista.cartagenamorros.com/pdf/41/10_CUIDADO_HUMANIZADO.pdf

8. Watson J. Watson's theory of humana caring and subjetive living experiences: carative factors/caritas peoccess as a disciplinary guide to the professional nursing practice. Texto Contexto Enferm [serie de internet]. Jan-mar 2007 [citado 12 jun 2016]; 16(1):129-135. Disponible en: http://www.scielo.br/pdf/tce/v16n1/a16v16n1.pdf

9. Alarcón SI. Necesidades espirituales de los pacientes terminales del servicio de medicina en el Hospital Nacional Dos de Mayo [tesis de licenciatura]. [internet]. Lima: Universidad Nacional de San Marcos; 2009 [citado 26 abr 2015]; p.97. Disponible en:

http://www.cybertesis.unmsm.edu.pe/bitstream/cybe rtesis/522/1/Alarcon_si.pdf
10. Bautista RLM, Parra CEE, Arias TKM, Parada OKG, Ascanio MKA, Villamarín CMU et al. Percepción de los comportamientos de cuidado humanizado en los usuarios hospitalizados en una institución de salud de $3^{\circ}$ y $4^{\circ}$ nivel de atención. Rev cienc cuidad [serie de internet]. 2015 [citado 24 set 2015]; 12(1):105-118. Disponible en: http://www.revistas.ufps.edu.co/ojs/index.php/cienci aycuidado/article/view/331/345

11. Miranda CP, Monje VP, Oyarzún GJ. Percepción de cuidado humanizado en el servicio de Medicina Adulto, Hospital Base Valdivia, tercer trimestre 2014 [tesis licenciatura]. [internet]. Valdivia: Universidad Austral de Chile; 2015. [citado 24 may 2016]. p. 62. Disponible en: http://www.cybertesis.uach.cl/tesis/uach/2014/fmm6 72p/doc/fmm672p.pdf

12. Borré OYM. Calidad Percibida de la Atención de Enfermería por pacientes hospitalizados en Institución Prestadora de Servicios de Salud en Barranquilla [tesis licenciatura]. [internet]. Barranquilla: Universidad Nacional de Colombia; 2013 [citado 12 ago 2014]; p.128. Disponible en http://www.bdigital.unal.edu.co/10553/1/539597.20 13.pdf

13. Silva-Fhon J, Ramón-Córdova S, VergaraVillanueva S, Palacios-Fhon V, PartezaniRodrigues A. Percepción del paciente hospitalizado respecto a la atención de enfermería en un hospital público. Enfermeria Universitaria [serie de internet]. Abr-jun 2015 [citado 09 agos 2016]; 12(2): 80-87. Disponible

en: http://www.redalyc.org/articulo.oa? id=35874184300 $\underline{5}$

14. Espinosa ML, Huerta BK, Pantoja QJ, Velásquez CW, Cubas CD, Ramos VA. El cuidado humanizado y la percepción del paciente en el Hospital EsSalud Huacho. Octubre de 2010. Ciencia y Desarrollo [serie de internet]. Ene-jun 2011. [citado 02 junio 2015]; 13(1): [cerca 09 laudas]. Disponible en: http://www.uap.edu.pe/investigaciones/Esp/Revista_ 13_Esp_05.pdf

15. Vanti M, El espíritu de San Camilo de Lellis. Italia; 1951, poligotta vaticana p.485, 284, 285-301.

16. Congreso de la República del Perú. Ley General de Salud. Ley $\mathrm{N}^{\mathrm{a}} 26842$ [internet]. Lima Perú: Congreso de la República; 1997 [citado 15 oct de 2015]. p.34. Disponible en: http://www.minsa.gob.pe/renhice/documentos/norm ativa/Ley26842-199720LeyGeneraldeSaludConcordada.pdf 
17. Rivera ALN, Triana A. Percepción de comportamientos de cuidado humanizado de enfermería en la Clínica Country. Av Enferm [serie de internet]. Ene-jun 2007 [citado 03 feb 2015]; 25(1): 56-68. Disponible: http://revistas.unal.edu.co/index.php/avenferm/articl e/view/35901/36916

18. Rivera ALN, Triana A. Cuidado humanizado de enfermería: visibilizando la teoría y la investigación en la práctica, en la Clínica del Country. Actual Enferm [serie de internet]. 2007 [citado $10 \mathrm{feb}$ 2015]; 10 (4): 15-21. Disponible en: https://www.ucursos.cl/medicina/2011/0/ENFENFCO24/1/noveda des/r/CUIDADO_HUMANIZADO_DE_ENFERME RIA_VISIBILIZANDO_LA_TEORIA_Y_LA_INV ESTIGACION_EN_LA_PRACTICA.pdf

19. Urra ME, Jana AA, García MV. Algunos aspectos esenciales del pensamiento de Jean Watson y su teoría de cuidados transpersonales. Cienc Enferm [serie de internet]. 2011 [citado 12 oct 2015]; 17(3): 11-22. Disponible en: http://www.scielo.cl/scielo.php?script=sci_arttext\&p $\underline{\mathrm{id}=\mathrm{S} 0717-95532011000300002}$

20. Brusco A. Humanización de la asistencia al enfermo. Madrid: Tres Cantos; 1998.

21. Zapata DSM. Percepción del paciente adulto mayor sobre el cuidado que recibe de la enfermera en el Servicio de Geriatría del Hospital Almenara [tesis de licenciatura]. [internet]. Lima Perú: Universidad Ricardo Palma; 2013 [citado 20 jun 105]. p.81. Disponible:

http://cybertesis.urp.edu.pe/bitstream/urp/311/1/Zap ata_sm.pdf

22. Romero ALC. Percepción del paciente acerca de la calidad de atención que brinda la enfermera en el servicio de medicina en el Hospital Nacional Daniel
Alcides Carrión [tesis para licenciatura]. [internet]. Lima-Perú: Universidad Nacional Mayor de San Marcos; 2008 [citado 23 mayo 2015]; p.68. Disponible en: http://cybertesis.unmsm.edu.pe/handle/cybertesis/48 $\underline{2}$

23. Bermejo J. Resiliencia, una mirada de humanización al sufrimiento. Madrid, PPC, 2011.

24. Pancrazzi R. El proceso de duelo. Roma, Italia: editorial Vaticano, 2014.

25. Gómez PR. La mística como experiencia analógica del ser. Franciscanum. Revista de las ciencias del espíritu, [serie de internet]. Ene-jun 2012 [citado 11 ago 2014 ]; 54(157): 273-306 Disponible en: http://www.redalyc.org/pdf/3435/343529078010.pdf

26. Romero ME, Contreras MIM, Moncada SA. Relación entre cuidado humanizado por enfermería con la hospitalización de pacientes. Hacia la promoción de la salud [serie de internet]. Ene-jun 2016 [citado 10 set 2016]; 21(1):26-36. Disponible en:

http://www.scielo.org.co/pdf/hpsal/v21n1/v21n1a03. pdf

27. Torres CCC. Percepción de la calidad del cuidado de enfermería en pacientes hospitalizados. Rev Enferm [serie de internet]. 2010 [citado 28 abr 2015]; 28(2): 92-110. Disponible en: http://revistas.unal.edu.co/index.php/avenferm/articl e/view/21385/22435

28. Sosa-Rosas MP, Cuamatsi-Peña MT. La mística de la profesión de enfermería. Rev Enferm Inst Mex Seguro Soc. [serie de internet]. 2015 [citado 30 ago 2015]; 23(1): 51-56. Disponible en: http://www.medigraphic.com/pdfs/enfermeriaimss/ei m-2015/eim151i.pdf 